\title{
Thermotropic Liquid Crystals Based on Chito-Oligosaccharides III. Effect of the Molecular Shape of N,O-Acylated Chito-Oligosaccharides on the Discotic Mesomorphism
}

\author{
Makoto Sugiura, Masahiko Minoda, Junji Watanabe,* \\ and Takeaki MiYAmoto \\ Institute for Chemical Research, Kyoto University, \\ Uji, Kyoto 611, Japan
}

(Received March 11, 1994)

\begin{abstract}
Acylated derivatives of chitosan oligomers with a relatively high degree of polymerization ( $D P=4,5$, or 6$)$ were synthesized and their mesophase properties were studied in comparison with those of the lower- $D P$ homologues $(D P=2$ and 3$)$ previously reported. By differential scanning calorimetry (DSC), all the compounds were found to show thermotropic mesomorphism in a temperature region below $150^{\circ} \mathrm{C}$. The isotropization temperatures decreased with increasing $D P$, while the melting point remained at around $65^{\circ} \mathrm{C}$, independent of $D P$. In addition to the narrowing of the mesomorphic region, the optical anisotropy as observed under a polarizing microscope remarkably decreased with increasing $D P$, indicating that the mesophase becomes less stable and ordered with an increase in DP. An X-ray diffraction analysis revealed that the tetramer as well as the dimer and trimer form a hexagonal ordered columnar phase $\left(D_{h o}\right)$, while the pentamer forms a disordered one $\left(D_{h d}\right)$. On the other hand, the phase formed by the hexamer was not a columnar but a discotic nematic phase $\left(N_{D}\right)$. This is attributable to the highly anisotropic shape of the oligosaccharide core in the hexamer.

KEY WORDS Chito-Oligosaccharide / Discotic Liquid Crystals / Degree of Polymerization (DP) / Anisotropic Molecular Shape / Hexagonal Columnar Phase /
\end{abstract}

The geometric shape of a mesogen is closely related to the mode of molecular arrangement in the liquid crystalline state, as is clearly observed in the structural difference between the calamitic mesophases formed by rigid-rod molecules and the discotic mesophases formed by disc-shaped molecules. In the discotic liquid crystals so far reported, ${ }^{1}$ the existence of an aromatic, rigid, flat and/or symmetric disc-like mesogen has been believed to be essential for generating some class of discotic mesophases. In fact, highly symmetric discogenic compounds often show a hexagonal-ordered discotic columnar phase $\left(\mathrm{D}_{\mathrm{ho}}\right)$, the most ordered structure in discotics known so far. However, it should be noted that a few compounds with a lower symmetry, e.g., a two-fold rotational symmetry can form, like disc-shaped molecules, a discotic columnar phase $\left(D_{h}\right)$. For example, alkyloxy substituted nitrophenylhydrazones with semi-discoidal molecular shape were found to induce a $D_{h}$ phase due to both the intramolecular hydrogen-bond formation and the intermolecular donor-acceptor interaction. ${ }^{2}$ We have also demonstrated that acylated cello- and chito-oligosaccharides can form an enantiotropic $D_{\text {ho }}$ phase. ${ }^{3-6}$ Compared to the mesophase of the cello-versions, those of the chito-oligosaccharide derivatives were characterized by an enhanced thermal stability, in

* On leave from Faculty of Engineering, Tokyo Institute of Technology, Ookayama, Meguro-ku, Tokyo 152, Japan. 
which the intermolecular hydrogen-bond interaction derived from the secondary amido functions at the $\mathrm{C}-2$ position of the repeating pyranose ring plays an important role.

Previous studies dealt with the chitobiose and chitotriose derivatives. ${ }^{5,6}$ In this study, we synthesized a series of acylated chito-oligosaccharides with a higher degree of polymerization $(D P=4,5$, or 6$)$. Because of the rigid and linear structure due to the $\beta-1,4$ linkage between the repeating units, the axial ratio of the chito-oligosaccharide core proportionally increases with an increase in $D P$. The increase in this axial ratio is expected to make the discotic mesophase less stable, compensating the strong intermolecular interaction and eventually rendering the discotic columnar phase utterly unstable. We examined the thermal properties and mesophase structure of these acylated chito-oligosaccharides to study their discogenic character and the stability of the mesophase.

\section{EXPERIMENTAL}

\section{Materials}

Chito-oligosaccharides (Yaizu Suisan Co., Ltd., Japan) were obtained as a hydrochloric acid salt (purity, 95-100\%) and employed without further purification. Acid chlorides (Tokyo Kasei Kogyo Co., Ltd., Japan) were used as received. Chloroform and pyridine (Nacalai Tesque, Inc., Japan) were distilled over calcium hydride prior to use. N,OAcylated chitotetraose, chitopentaose and chitohexaose (Figure 1) will be abbreviated as CHTmAn $(m=4-6, n=10-18)$, in which $m$ refers to the degree of polymerization and $n$ to the total number of the carbon atoms in the substituents.

Acylation of chito-oligosaccharides was carried out by reacting them with a long-alkyl fatty acid chloride in the presence of a base according to the previously reported procedure $^{5}$ with some modifications made to cope with the decreased reactivity and solubility of

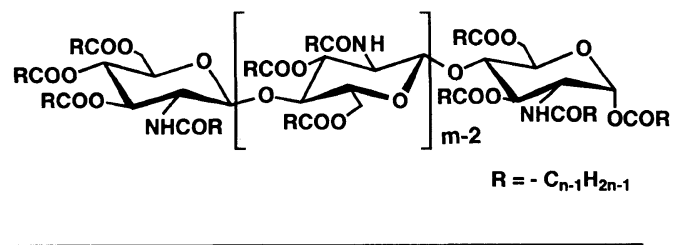

\begin{tabular}{cll}
\hline $\begin{array}{c}\text { Degree of } \\
\text { polymerizatiom } \\
(\mathrm{m})\end{array}$ & Sample code & \multicolumn{1}{c}{$n$} \\
\hline 2 & CHT2An & $10,12,14,18$ \\
3 & CHT3An & $10,12,14,18$ \\
4 & CHT4An & $10,12,14,18$ \\
5 & CHT5An & $10,14,18$ \\
6 & CHT6An & 14, \\
\hline
\end{tabular}

Figure 1. Structures of acylated chito-oligosaccharides.

the chito-oligosaccharides with larger $D P$.

\section{Methods}

In a typical preparation, chitopentaose hydrochloride $(80 \mathrm{mg} ; 97.1 \mathrm{mmol})$ was soaked in pyridine $(5 \mathrm{ml})$ for $1 \mathrm{~h}$ and then the pyridine was evaporated off under a reduced pressure. The liberated chitopentaose was dispersed in a mixture of pyridine $(5 \mathrm{ml})$, dimethylaminopyridine $(2.0 \mathrm{~g})$, and chloroform $(30 \mathrm{ml})$ with a magnetic stirring under nitrogen atmosphere. To this solution, myristoyl chloride $(1.35 \mathrm{ml}$; 50 equivalents to the chitopentaose) was added dropwise at room temperature in the dark. The suspension was stirred for $18 \mathrm{~h}$ at $40^{\circ} \mathrm{C}$. The reaction mixture changed from a heterogeneous colorless solution to a homogeneous orange solution. After the solution was cooled and concentrated to $c a .10 \mathrm{ml}$ under a reduced pressure, it was poured into methanol to remove the excess acid chloride, and the precipitate was purified by reprecipitation with a chloroform/methanol system and dried in vacuo overnight. Other derivatives were prepared in the same manner, excepting that the synthesis of CHT6A14 required a more moderate condition to suppress the formation of the undesirable $N, N$-diacylated by-product. The overall yield decreased with an increase in DP: it was ca. $40 \%$ for CHT4Ans; ca. $15 \%$ for CHT5Ans; $c a$. $10 \%$ for CHT6A14. All of the derivatives were white or slightly yellow 
powder and soluble in common organic solvents such as chloroform, benzene, and tetrahydrofuran.

\section{Measurement}

Infrared (IR) spectra were obtained with a JASCO FT/IR spectrometer Model 8800, Japan, by regulating the sample temperature with a Mettler Model FP-82 hot-stage, Switzerland. ${ }^{1} \mathrm{H}$ NMR spectra were recorded in $\mathrm{CDCl}_{3}$ on a Varian VXR-200 $(200 \mathrm{MHz})$ instrument, the United States. Thermal phase behavior was studied by differential scanning calorimetry (DSC) with a Rigaku Denki Model DSC-8230, Japan, at a constant heating/ cooling rate of $10^{\circ} \mathrm{C} \mathrm{min}^{-1}$. Thermal optical microscopy was performed on a Nikon Model Optiphoto-Pol, Japan, employing a Mettler Model FP-82 hot-stage. X-Ray diffraction patterns of the samples in a glass capillary were recorded at different temperatures, using $\mathrm{Ni}$ filtered $\mathrm{Cu}-K_{\alpha}$ radiation. The sample temperatures were regulated by use of a Mettler Model FP-80 hot-stage.

\section{RESULTS AND DISCUSSION}

\section{Synthesis of N,O-Acylated Chito-Oligosac- charides}

A series of chito-oligosaccharide alkanoates having different $D P$ were synthesized. IR and ${ }^{1} \mathrm{H}$ NMR analyses revealed that the obtained products possess the expected structure in which the amino groups of the starting chito-oligosaccharides were $N$-monoacylated (not $N, N$-diacylated). For example, the ${ }^{1} \mathrm{H}$ NMR spectra of the acylated chitotetraose, gave an overlapped absorption $(\delta=5.65-5.95)$ of the $\mathrm{N}^{\prime}-\mathrm{H}, \mathrm{N}^{\prime \prime}-\mathrm{H}$, and $\mathrm{N}^{\prime \prime \prime}-\mathrm{H}$ groups together with a doublet peak $(\delta=5.55)$ of the $\mathrm{N}-\mathrm{H}$ group in the reducing monomer unit. In addition, a doublet peak at $\delta=6.10$ assignable to the $\alpha$-anomeric proton predominated, showing an excellent stereoselectivity of the present acylation. The results of elemental analysis are listed in Table I. The observed elemental contents were in good agreement with those calculated for the expected structure, in all cases. Thus the tetra-, penta-, and hexa-oligosaccharide derivatives possess 14, 17, and 20 acyl pendants, respectively. This was also confirmed by ${ }^{1} \mathrm{H}$ NMR. Their purity was further checked by size exclusion chromatography (purity $\sim 100 \%$ ).

\section{Thermal Properties}

To investigate the thermal properties, differential scanning calorimetric (DSC) measurements were carried out. Like the dimer and trimer counterparts, ${ }^{5}$ all of the compounds

Table I. Characterization of the acylation products

\begin{tabular}{|c|c|c|c|c|c|c|}
\hline \multirow{3}{*}{ Sample } & \multirow{3}{*}{$D P$} & \multirow{3}{*}{$\begin{array}{l}\text { Molecular } \\
\text { weight }^{\mathrm{a}}\end{array}$} & \multirow{3}{*}{$\begin{array}{l}\text { Molecular } \\
\text { Formula }\end{array}$} & \multicolumn{3}{|c|}{ Elemental analysis } \\
\hline & & & & $\% \mathrm{C}$ & $\% \mathrm{H}$ & $\% \mathrm{~N}$ \\
\hline & & & & \multicolumn{3}{|c|}{ Found (Calcd) } \\
\hline CHT4A10 & 4 & $2.82 \times 10^{3}$ & $\mathrm{C}_{164} \mathrm{H}_{298} \mathrm{~N}_{4} \mathrm{O}_{31}$ & $69.55(69.80)$ & $10.34(10.64)$ & $1.96(1.99)$ \\
\hline CHT4A12 & 4 & $3.22 \times 10^{3}$ & $\mathrm{C}_{192} \mathrm{H}_{354} \mathrm{~N}_{4} \mathrm{O}_{31}$ & $71.99(71.73)$ & $11.24(11.10)$ & $1.68(1.74)$ \\
\hline CHT4A14 & 4 & $3.60 \times 10^{3}$ & $\mathrm{C}_{220} \mathrm{H}_{410} \mathrm{~N}_{4} \mathrm{O}_{31}$ & $72.97(73.24)$ & $11.45(11.45)$ & $1.50(1.55)$ \\
\hline CHT4A18 & 4 & $4.39 \times 10^{3}$ & $\mathrm{C}_{276} \mathrm{H}_{522} \mathrm{~N}_{4} \mathrm{O}_{31}$ & $75.88(75.46)$ & $12.33(11.98)$ & $1.32(1.28)$ \\
\hline CHT5A10 & 5 & $3.44 \times 10^{3}$ & $\mathrm{C}_{200} \mathrm{H}_{363} \mathrm{~N}_{5} \mathrm{O}_{38}$ & $69.15(69.71)$ & $10.45(10.62)$ & $2.02(2.03)$ \\
\hline CHT5A14 & 5 & $4.40 \times 10^{3}$ & $\mathrm{C}_{268} \mathrm{H}_{499} \mathrm{~N}_{5} \mathrm{O}_{38}$ & $73.42(73.16)$ & $11.15(11.43)$ & $1.57(1.59)$ \\
\hline CHT5A18 & 5 & $5.35 \times 10^{3}$ & $\mathrm{C}_{336} \mathrm{H}_{635} \mathrm{~N}_{5} \mathrm{O}_{38}$ & $75.11(75.38)$ & $11.68(11.96)$ & $1.31(1.31)$ \\
\hline CHT6A14 & 6 & $5.19 \times 10^{3}$ & $\mathrm{C}_{316} \mathrm{H}_{588} \mathrm{~N}_{6} \mathrm{O}_{45}$ & $72.93(73.10)$ & $11.23(11.41)$ & $1.64(1.62)$ \\
\hline
\end{tabular}

\footnotetext{
a Calculated from the molecular formula.
} 


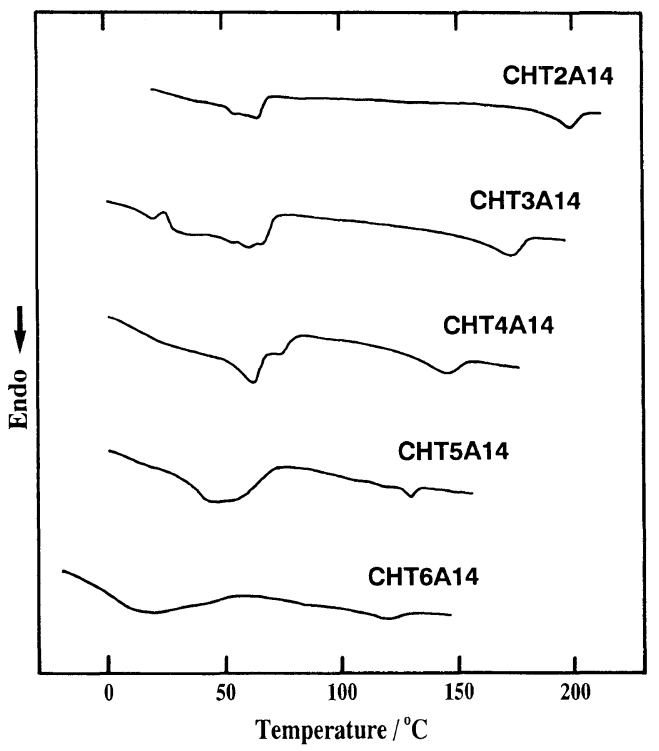

Figure 2. Heating DSC traces of CHT $m \mathrm{Al} 14(m=2-6)$.

Table II. Transition temperatures $T_{\mathrm{m}}$ and $T_{\mathrm{i}}$ and enthalpy changes at $T_{\mathrm{i}}(\Delta H)$ obtained for CHT4An, CHT5A $n$, and CHT6A14

\begin{tabular}{ccccc}
\hline & \multicolumn{2}{c}{ Transition temp ${ }^{\mathrm{a}} /{ }^{\circ} \mathrm{C}$} & & \multicolumn{1}{c}{$\Delta H$} \\
\cline { 2 - 3 } \cline { 5 - 5 } Product & $T_{\mathrm{m}}$ & $T_{\mathrm{i}}$ & & $\mathrm{kcal} / \mathrm{mru}$ \\
\hline CHT4A10 & 53 & 160 & & 4.20 \\
CHT4A12 & 56 & 152 & & 1.00 \\
CHT4A14 & 76 & 145 & & 0.75 \\
CHT4A18 & 77 & 143 & & 2.43 \\
CHT5A10 & 61 & 140 & & 0.65 \\
CHT5A14 & 52 & 130 & & 0.25 \\
CHT5A18 & 65 & 120 & & 0.45 \\
CHT6A14 & $40^{\mathrm{b}}$ & 120 & & 0.36 \\
\hline
\end{tabular}

a Determined by DSC.

b Determined by microscopic observation under a polarizing microscope.

prepared here reproducibly showed two transition peaks both in heating and cooling mode, indicating the induction of an enantiotropic mesomorphism. The representative heating DSC traces of CHTmA14 $(m=2-6)$ are presented in Figure 2. The transition temperatures and enthalpies obtained in the heating mode are listed in Table II. Since CHT6A14, which has largest oligosaccharide core, showed

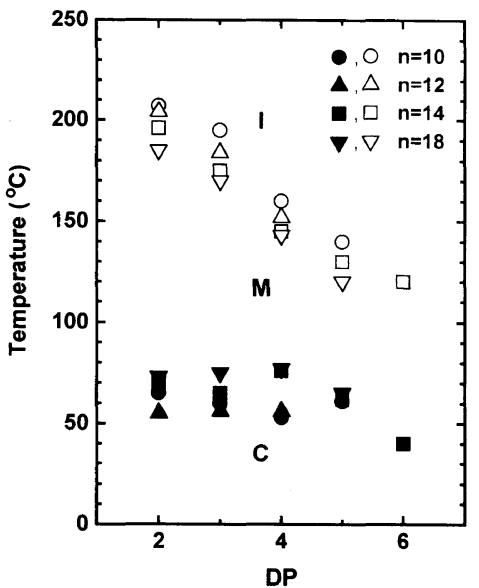

Figure 3. Transition temperatures as a function of the degree of polymerization $(D P)$ : Open symbols, isotropization point $\left(T_{\mathrm{i}}\right)$; closed symbols $\left(T_{\mathrm{m}}\right) . \mathrm{I}, \mathrm{M}$, and $\mathrm{C}$ stand for the isotropic phase, mesophase and crystalline phase, respectively.

no distinct transition from the crystal to the mesophase, microscopic observations under a polarizing microscope were instead performed to determine the transition temperature. The $\Delta H$ values at the isotropization point $T_{\mathrm{i}}$ of CHT4Ans were comparable to those of the lower- $D P$ derivatives $(2.6-4.2 \mathrm{kcal} / \mathrm{mru}$ for the dimers and $1.5 \sim 4.8 \mathrm{kcal} / \mathrm{mru}$ for the trimers $\left.{ }^{5}\right)$. However, much smaller $\Delta H$ values were obtained for CHT5Ans and CHT6A14. No systematic dependence of $\Delta H$ on the pendant length was observed for the series of derivatives having common $D P$, whereas the isotropization temperature slightly decreased with an increase in the pendant length.

The relationships, between the transition temperature and $D P$ are summarized in Figure 3. Irrespective of the pendant length, the isotropization temperature decreases with increasing $D P$, while the melting point remains constant at around $65^{\circ} \mathrm{C}$. Thus, an increase in $D P$ brings about a narrowing of the mesophase temperature range.

The textures and optical anisotropy of the mesophases observed under a polarizing microscope also gave a similar indication. The 
leaf-like texture, which is typical of highly ordered discotic columnar phases, was clearly observed for CHT2An $(D P=2)^{5}$ but with increasing $D P$, the texture became less and less clear, and finally, CHT6A14 showed little birefringence. Thus an increase in shape anisotropy of the molecules reduces the stability and the degree of order of the mesophase. A similar result has been reported for acylated cello-oligosaccharides. ${ }^{8}$

In Figure 4, the DP dependence of the transition temperatures of the acylated chitooligosaccharides is compared with that of the cello-versions, ${ }^{8}$ in which the pendant length is fixed at $n=10$. Both series of the derivatives have a similar structure except for the substituent at the C-2 position. However, this small structural difference brings about a surprisingly large difference in the thermal stability of the mesophase: CHTmA10s possess a mesophase in a much wider range of temperature than the cello-versions (CELmA10s). For example, the chito-pentamer (CHT5A10) exhibits a mesophase from about $60^{\circ} \mathrm{C}$ up to as high as $140^{\circ} \mathrm{C}$, while the cello-pentamer (CEL5A10) has virtually no mesophase. These results demonstrate that the intermolecular

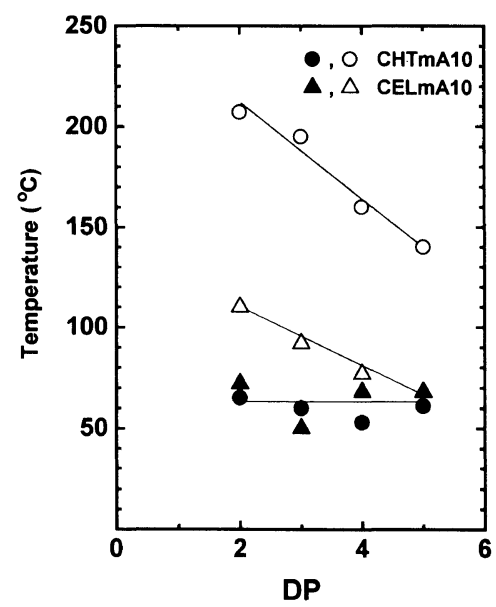

Figure 4. The $D P$ dependence of the transition temperatures for chito-oligosaccharide $(\bigcirc, 0)$ and cellooligosaccharide derivatives $(\triangle, \boldsymbol{\Delta}): \bigcirc$ and $\triangle$, isotropization point; $\boldsymbol{O}$ and $\boldsymbol{\Delta}$, melting point. hydrogen-bonding in the chito-derivatives makes a large contribution to the formation of the stable mesophases. The mesophases induced by chito-oligosaccharides are presumed to be analogous to the ones formed by the cello-versions, i.e., discotic columnar phases. $^{3,4}$

\section{Mesophase Structures}

By X-ray diffraction analysis, the mesophase structures of CHT4A $n$, CHT5A $n$, and CHT6A14 were determined precisely. The spacings corresponding to the observed reflections are listed in Table III. The diffraction patterns of CHT4A $n$ specimen contain one strong and two or three weak diffraction rings in the small angle region together with a sharp one in the wide angle region. When oriented by shearing, all reflections at small angles lie on the equatorial line perpendicular to the shearing direction, while the reflection at wide angles appears on the meridional line. In the case of CHT4A14, for example, peaks at small angles, which are indexed as $h k 0$, correspond to real spacings of $31.8 \AA, 18.5 \AA, 15.7 \AA$, and $11.9 \AA$, respectively, the ratios being very close to $1: 1 / 3^{1 / 2}: 1 / 2: 1 / 7^{1 / 2}$. This result indicates a hexagonal symmetry of the column packing, as in the case of the dimeric (CHT2A $n$ ) and the trimeric (CHT3An) counterparts. ${ }^{6}$ The same packing features were found also for CHT4A $n$ and CHT5A $n$ even though the intensities of the 110 reflection for them are weaker than those for CHT2Ans and CHT3Ans. On the other hand, CHT6A14 showed only an ill-defined broad halo at small angles, meaning the lack of columnar structure in this derivative.

In Figure 5a, the lattice spacing of the 100 reflection, which can be considered as the diameter of the column, is plotted against the side chain length $n$. An increase in the 100 spacing in proportion to $n$ is observed for both CHT4Ans and CHT5Ans as in the case of CHT2Ans and CHT3Ans. ${ }^{6}$ In addition, the 100 spacing increases with increasing $D P$ for a 
Table III. X-Ray diffraction data and lattice parameters obtained for the mesophase of CHT4A $n$, CHT5A $n$, and CHT6A14

\begin{tabular}{|c|c|c|c|c|c|c|}
\hline \multirow{2}{*}{ Sample } & \multirow{2}{*}{$\frac{\text { Temp }}{{ }^{\circ} \mathrm{C}}$} & \multirow{2}{*}{$\frac{d_{\mathrm{obs}}}{\AA}$} & \multirow{2}{*}{$\frac{d_{\text {calcd }}{ }^{\mathrm{a}}}{\AA}$} & \multirow{2}{*}{ Index } & \multirow{2}{*}{ Phase } & \multirow{2}{*}{$\frac{\text { Lattice parameter }}{\AA}$} \\
\hline & & & & & & \\
\hline \multirow[t]{3}{*}{ CHT4A10 } & 100 & 26.3 (s) & 26.4 & 100 & $\mathrm{D}_{\text {ho }}$ & $a=30.5$ \\
\hline & & $13.2(\mathrm{~m})$ & 13.2 & 200 & & \\
\hline & & $5.15(w)$ & & 001 & & \\
\hline \multirow[t]{5}{*}{ CHT4A14 } & 100 & $31.8 \quad(\mathrm{~s})$ & 31.9 & 100 & $\mathrm{D}_{\text {ho }}$ & $a=36.8$ \\
\hline & & 18.5 (w) & 18.4 & 110 & & \\
\hline & & $15.7(\mathrm{~m})$ & 15.9 & 200 & & \\
\hline & & $11.9(\mathrm{~m})$ & 12.0 & 210 & & \\
\hline & & $5.18(w)$ & & 001 & & \\
\hline \multirow[t]{5}{*}{ CHT4A18 } & 100 & $35.6 \quad(s)$ & 35.2 & 100 & $\mathrm{D}_{\text {ho }}$ & $a=40.7$ \\
\hline & & 20.2 (w) & 20.3 & 110 & & \\
\hline & & $17.6(\mathrm{~m})$ & 17.6 & 200 & & \\
\hline & & $13.6(\mathrm{w})$ & 13.3 & 210 & & \\
\hline & & $4.96(\mathrm{vw})$ & & 001 & & \\
\hline \multirow[t]{2}{*}{ CHT5A10 } & 100 & $28.0 \quad(\mathrm{~s})$ & 28.1 & 100 & $D_{h d}$ & $a=32.4$ \\
\hline & & $13.9(w)$ & 14.0 & 200 & & \\
\hline \multirow[t]{3}{*}{ CHT5A14 } & 90 & $33.6 \quad(\mathrm{~s})$ & 33.6 & 100 & $\mathrm{D}_{\mathrm{hd}}$ & $a=38.8$ \\
\hline & & $17.0 \quad(w)$ & 16.8 & 200 & & \\
\hline & & 12.4 (vw) & 12.6 & 210 & & \\
\hline \multirow[t]{3}{*}{ CHT5A18 } & 90 & $38.1 \quad(\mathrm{~s})$ & 38.1 & 100 & $\mathrm{D}_{\mathrm{hd}}$ & $a=44.0$ \\
\hline & & $18.8(\mathrm{~m})$ & 19.0 & 200 & & \\
\hline & & $14.4(w)$ & 14.4 & 210 & & \\
\hline
\end{tabular}

a Calculation based on the lattice parameters obtained for the two-dimensional hexagonal lattice.

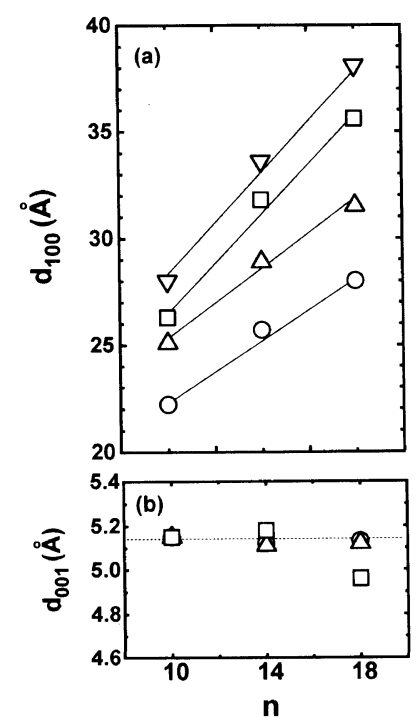

Figure 5. Plots of the lattice spacing of (a) 100 reflection and (b) 001 reflection versus the total number, $n$, of carbon in the acyl pendant: $\bigcirc, \mathrm{CHT} 2 \mathrm{~A} n ; \triangle, \mathrm{CHT} 3 \mathrm{~A} n ; \square$, CHT4A $n ; \nabla$, CHT5A $n$. fixed value of $n$.

The X-ray pattern of the CHT4A $n$ specimen includes a sharp reflection, indexed as 001 , at wide angles, indicating an ordered stacking of molecules within a column. The lattice spacing of this reflection, $d_{001}$, coincides with the intermolecular distance along the column axis. Figure $5 \mathrm{~b}$ depicts the relationship between $d_{001}$ and $\mathrm{n}$ for CHT4A $n$ together with those for CHT2A $n$ and CHT3A $n$. The $d_{001}$ values for CHT4A10 and CHT4A14 are nearly equal to the constant value of $c a .5 .15 \AA$ obtained for the dimeric and the trimeric analogues, whereas that for CHT4A18 is slightly smaller. The lattice parameters $a$ obtained for the most plausible hexagonal lattice for CHT4A $n$ are summarized in Table III. The spacings calculated with this lattice parameters are in good agreement with the observed values (Table III). A further support for these lattices 
can be obtained from a density consideration: Assuming that each molecule is included in a unit lattice volume, we calculate the specific gravities to be $0.9-1.0 \mathrm{~g} \mathrm{ml}^{-1}$ for the CHT4An mesophases. These values are very plausible in light of those for the hexagonal ordered columnar mesophases of similar chemical compositions thus far reported. ${ }^{5,6}$ On the other hand, no 001 reflection can be observed for CHT5An, showing a disordered molecular stacking in the columns. As described above, the isotropization enthalpies $\Delta H$ of CHT5Ans are much smaller than those of CHT4Ans, which can be attributable to the less ordered mesophase structure.

The typical X-ray diffraction patterns and the possible structures of the mesophases for the tetrameric, pentameric, and hexameric alkanoates are illustrated in Figure 6. The phases of CHT4A $n$ and CHT5A $n$ are both assignable to a hexagonal columnar phase in a class of discotics; furthermore, the former is distinguished from the latter by the presence of the ordered molecular stacking along the column axis. According to Destrade's terminology, ${ }^{7}$ the mesophase of CHT4A $n$ corresponds to a $\mathrm{D}_{\text {ho }}$ phase while CHT5A $n$ to a $\mathrm{D}_{\mathrm{hd}}$ phase. The hexameric alkanoate CHT6A14 exhibits no discotic columnar mesomorphism.

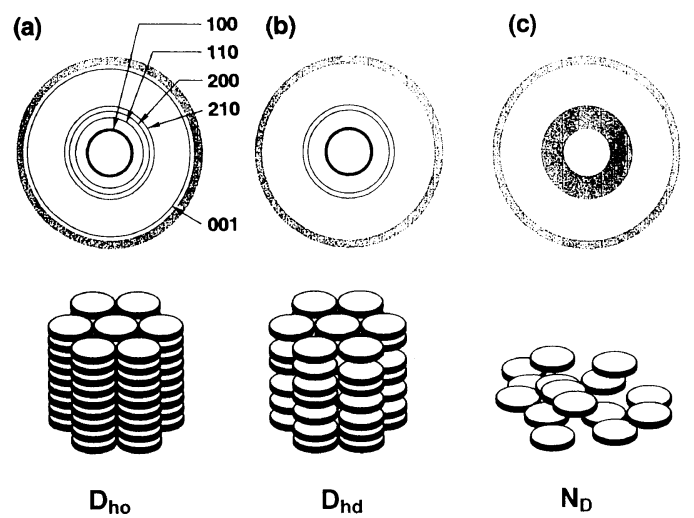

Figure 6. The typical X-ray diffraction patterns (upper) and the possible structures (lower) of the discotic mesophases: (a) $\mathrm{D}_{\text {ho }}$ for CHT2An, CHT3A $n$, and CHT4A $n$, (b) $\mathrm{D}_{\text {hd }}$ for CHT5An, and (c) $\mathrm{N}_{\mathrm{D}}$ for CHT6A14.
The phase of this derivative is believed to be a discotic nematic $\left(\mathrm{N}_{\mathrm{D}}\right)$. This difference presumably results from the difference in the axial ratios of the core saccharides; consequently, chitopentaose possesses the maximum axial ratio so as to behave as a discotic columnar mesogen. The intermolecular hydrogen-bonding is responsible for the enhanced stability of the chito-derivatives compared to that of the cello-counterparts.

Supporting evidence for the contribution of the secondary amido groups to the stabilization of the mesophase comes from the infrared (IR) measurement conducted with regulating the sample temperature by a hot stage instrument. Here the pendant length is fixed at $n=14$ with $D P$ ranging from 2 to 6 . In the IR spectra of the acylated chito-oligosaccharides, $\mathrm{N}-\mathrm{H}$ stretching band of the secondary amido group appears at around $3300 \mathrm{~cm}^{-1}$, the variation of which band is a measure of the strength of the hydrogen-bonding interaction. Measurement was performed after 10 minutes aging at $2.5-10{ }^{\circ} \mathrm{C}$ intervals in a wide temperature range covering the crystalline, liquid crystalline, and isotropic liquid states. In Figure 7, the frequency of the amido absorption band was plotted against temperature.

In the crystalline and liquid crystalline states, the absorption frequency remains almost independent of temperature for all compounds.

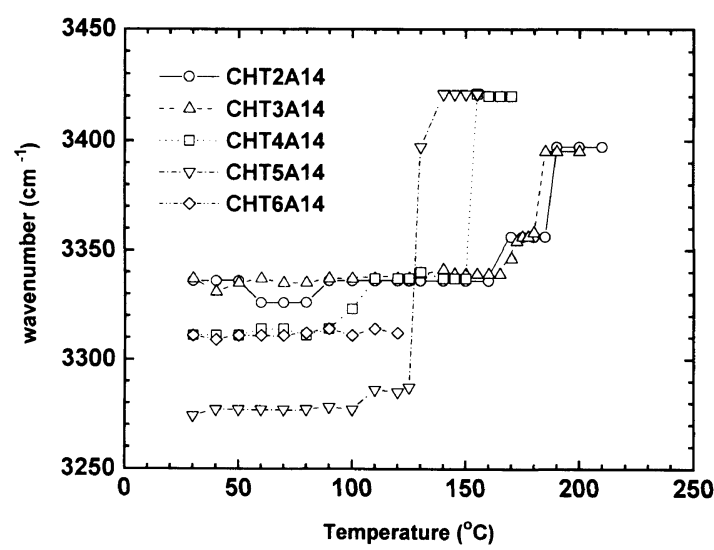

Figure 7. Temperature dependence of the frequency of the $\mathrm{N}-\mathrm{H}$ stretching band for the indicated specimens. 
Considerable frequency differences are seen among different samples in the crystalline and liquid crystalline phases, but no systematic relationship between the absorption frequency and $D P$ can be obtained. At the transition from the mesophase to the isotropic phase, the $\mathrm{N}-\mathrm{H}$ absorption peak for the derivatives with $D P$ of 2 to 5 is drastically shifted to higher frequencies above $3400 \mathrm{~cm}^{-1}$, indicating that the intermolecular hydrogen-bonding relevant to the secondary amido groups either disappears or is remarkably weakened in the isotropic melt. In contrast to this, in the case of CHT6A14, which does not form a columnar structure, the peak is not shifted at the clearing point but just broadens and becomes hardly detectable. This confirms that the mesophase transition behavior of CHT6A14 is fundamentally different from those of the lower- $D P$ derivatives.

In conclusion, the chitotetraose- and chitopentaose alkanoates can work as a discotic mesogen, forming a highly ordered discotic columnar phase, $D_{\text {ho }}$ and $D_{h d}$. The hexameric derivatives do not form a columnar phase but a discotic nematic phase. The result shows that the chitopentaose possesses the maximum axial ratio so as to behave as a discotic mesogen, although the fact that the columns built up by such fairly asymmetric molecules are packed in a highly symmetric lattice, i.e., a hexagonal lattice may be surprising and poses an interesting question as to the fashion of molecular packing within each column. Compared with cello-oligosaccharide counterparts, the mesophase temperature range of chitoversions is remarkably expanded mainly due to the increase in $T_{\mathrm{i}}$ temperature. Furthermore, the stacking period along the column, $d_{001}$, decreased. These can be interpreted as resulting from the intermolecular hydrogen-bonding which is formed between the secondary amido group in the C-2 position and the ester group.

Acknowledgments. We are indebted to Yaizu Suisan Co., Ltd. for supplying chitooligosaccharides.

\section{REFERENCES}

1. For a review, see: S. Chandrasekhar and G. S. Ranganath, Rep. Prog. Phys., 53, 57 (1990).

2. W. Paulus, H. Ringsdorf, S. Diele, and G. Pelzl, Liq. Cryst., 9, 807 (1991).

3. T. Itoh, A. Takada, T. Fukuda, T. Miyamoto, Y. Yakoh, and J. Watanabe, Liq. Cryst., 9, 221 (1991).

4. A. Takada, T. Fukuda, T. Miyamoto, Y. Yakoh, and J. Watanabe, Liq. Cryst., 12, 337 (1992).

5. M. Sugiura, M. Minoda, J. Watanabe, T. Fukuda, and T. Miyamoto, Bull. Chem. Soc. Jpn., 65, 1939 (1992).

6. M. Sugiura, M. Minoda, J. Watanabe, T. Fukuda, and T. Miyamoto, Liq. Cryst., 12, 603 (1992).

7. A. M. Levelut, J. Malthete, C. Destrade, and N. H. Tinh, Liq. Cryst., 2, 877 (1987).

8. A. Takada, K. Fujii, J. Watanabe, T. Fukuda, and T. Miyamoto, Macromolecules, 27, 1651 (1994). 